\title{
Vertex renormalization in DC conductivity of doped chiral graphene
}

\author{
E. Cappelluti ${ }^{1,2}$ and L. Benfatto ${ }^{3,1,2}$ \\ ${ }^{1}$ SMC Research Center, CNR-INFM, and ISC-CNR, via dei Taurini 19, 00185 Roma, Italy \\ ${ }^{2}$ Dipart. di Fisica, Università "La Sapienza", P.le A. Moro 2, 00185 Roma, Italy and \\ ${ }^{3}$ Centro Studi e Ricerche "Enrico Fermi", via Panisperna 89/A, I-00184, Rome, Italy
}

(Dated: October 30, 2018)

\begin{abstract}
The remarkable transport properties of graphene follow not only from the the Dirac-like energy dispersion, but also from the chiral nature of its excitations, which makes unclear the limits of applicability of the standard semiclassical Boltzmann approach. In this paper we provide a quantum derivation of the transport scattering time in graphene in the case of electron-phonon interaction. By using the Kubo formalism, we compute explicitly the vertex corrections to the DC conductivity by retaining the full chiral matrix structure of graphene. We show that at least in the regime of large chemical potential the Boltzmann picture is justified. This result is also robust against a small sublattice inequivalence, which partly spoils the role of chirality and leads to a doping dependence of the resistivity that can be relevant to recent transport experiments in doped graphene samples.
\end{abstract}

PACS numbers: 72.10.Di,63.20.kd,81.05.Uw

\section{INTRODUCTION}

The physical properties of doped and undoped graphene are the subject of an intense investigation in the view of possible applications of these materials in electronic and optical devices. A precise characterization of its transport and optical properties is thus a compelling issue. In addition to its relevance for technological applications, graphene poses several unusual and interesting theoretical problems. The low energy properties are dominated by the Dirac-like excitations at the so-called $\mathrm{K}$ and $\mathrm{K}^{\prime}$ points of the Brillouin zone, where the tightbinding electronic dispersion of the graphene honeycomb lattice can be approximated as $\epsilon_{\mathbf{k}}= \pm \hbar v_{\mathrm{F}}|\mathbf{k}|$. Here $\mathbf{k}$ is the relative momentum with respect to the $\mathrm{K}\left(\mathrm{K}^{\prime}\right)$ point $1 \underline{1}$ An additional ingredient is also the chiral character of the bands, which gives rise to the well-known absence of backward scattering in transport $\stackrel{2}{2}$ Note that, although obviously related in graphene, these two issues are formally distinct and are associated with different phenomenologies. A Dirac-like behavior can be induced for instance in nodal $d$-wave superconductors, as cuprates, without any relation to chirality $\frac{3.4,5}{4}$ On the other hand a chiral structure is still present even in bilayer graphene, where the low energy bands acquire a parabolic character $\underline{\underline{6}}$

Transport properties represent a particular delicate issue in graphene. As in any other system, also for graphene the full quantum treatment of transport processes would require the explicit evaluation of the current vertex corrections for the conductivity $\underline{\underline{7}, 8}$ Indeed, only going beyond the so-called bare-bubble approximation one can recover the distinction between the transport relaxation time $\tau_{\mathrm{tr}}$ and the "quasi-particle" one $\tau_{\mathrm{qp}}$ (relevant for instance for photoemission measurements). However, the chiral nature of excitations in graphene increases considerably the complexity of this approach. For this reason, transport properties are often discussed in doped graphene (i.e. when the chemical potential is far from the Dirac point) within the framework of the semiclassical Boltzmann theory $9.10,11,12,13,14,15,16,17$ In this context, $\tau_{\mathrm{tr}}$ differs from $\tau_{\mathrm{qp}}$ for a weighted average of the scattering probability with the angular factor $(1-\cos \theta)$ (where $\theta$ is the angle between incoming and outcoming scattering electrons). As usual, this leads to the suppression of forward scattering processes $\underline{18,19}$

Even though a general expectation holds that Boltzmann theory should be valid for doped graphene, the application of this approach to a chiral system as graphene is far from being trivial. Indeed, due to the multiband chiral structure, the velocity operator $\hat{\mathbf{v}}_{\mathbf{k}}=\hbar^{-1} d \hat{H}_{\mathbf{k}} / d \mathbf{k}$ in graphene does not commute with the Hamiltonian $\hat{H}_{\mathbf{k}}=\hbar v_{\mathrm{F}} \mathbf{k} \cdot \boldsymbol{\sigma}$ itself, where $\mathbf{k}=\left(k_{x}, k_{y}\right)$ and $\boldsymbol{\sigma}=\left(\hat{\sigma}_{x}, \hat{\sigma}_{y}\right)$ is the vector of the Pauli matrices. As it has been observed by many authors $, 20,21,22$ this fact poses several doubts on the applicability of conventional Boltzmann theory. In Boltzmann theory indeed one assumes that the equilibrium distribution function $f\left(\epsilon_{\mathbf{k}}\right)$ in the presence of the external electric field $\mathbf{E}$ can be described in terms of the one in the absence of external fields as $f_{\mathbf{E}}(\mathbf{k}) \approx f^{0}\left(\epsilon_{\mathbf{k}}-e \tau_{\mathrm{tr}} \mathbf{v}_{\mathbf{k}} \cdot \mathbf{E}\right)$, where $\mathbf{v}_{\mathbf{k}}=\hbar^{-1} d \epsilon_{\mathbf{k}} / d \mathbf{k}$ and $e$ is the electron charge ${ }^{23}$ An important underlying assumption here is that the energy eigenvalue $\epsilon_{\mathbf{k}}$ is a good quantum number as well as the shifted quantity $\epsilon_{\mathbf{k}}-e \tau_{\mathrm{tr}} \mathbf{v}_{\mathbf{k}} \cdot \mathbf{E}$. This would imply that the energy Hamiltonian operator $\hat{H}_{\mathbf{k}}$ and the velocity operator $\hat{\mathbf{v}}_{\mathbf{k}}$ commute so that they can be diagonalized simultaneously. As discussed above, however, this condition is not fulfilled in chiral graphene. To overcome this problem alternative approaches based on quantum and/or quasiclassical kinetic equations have been employed, where distribution functions and the corresponding density operators are defined in a chiral matrix space $:^{20,21,22}$

A second potential limit in the applicability of Boltzmann theory concerns the origin of the angular factor $(1-\cos \theta)$ in the expression of the transport scattering time. Indeed, in conventional systems it originates from the momentum dependence of the current operator 
$\hat{\boldsymbol{j}}(\mathbf{k})=(e / \hbar) d \hat{H}_{\mathbf{k}} / d \mathbf{k}$, which points along the $\mathbf{k}$ direction in the isotropic case, $\hat{\boldsymbol{j}}(\mathbf{k}) \propto \mathbf{k} /|\mathbf{k}|$. It is precisely such directional dependence which gives rise to the angular factor $1-\mathbf{v}_{\mathbf{k}} \cdot \mathbf{v}_{\mathbf{k}^{\prime}} /\left|\mathbf{v}_{\mathbf{k}}^{2}\right| \approx 1-\mathbf{k} \cdot \mathbf{k}^{\prime} /\left|\mathbf{k}^{2}\right|=1-\cos \theta$ in the transport properties $\frac{7.8}{1.8}$ Things are drastically different in the case of graphene where we have

$$
\hat{\boldsymbol{j}}(\mathbf{k})=\frac{e}{\hbar} \frac{d \hat{H}_{\mathbf{k}}}{d \mathbf{k}}=e v_{\mathrm{F}} \boldsymbol{\sigma} .
$$

The important feature to be stressed is that the bare vertex current operator is here $\mathbf{k}$-independent, i.e. it does not depend on the direction of the momentum $\mathbf{k}=$ $\left(k_{x}, k_{y}\right)$. Thus the possible relevance of the angular factor $(1-\cos \theta)$ suppressing forward scattering in the transport properties should be better assessed. This is particularly important when the scattering processes involve a momentum dependence more complex that the short-range impurity scattering discussed in Refs. 24, 25,26. A typical example is provided by the scattering of electrons by acoustic phonons, which plays a major role in controlling the temperature dependence of the DC conductivity $\underline{27,28}$

In order to assess these open issues we provide in this paper an explicit derivation of the DC conductivity in doped graphene using a fully quantum approach for the electron-phonon scattering based on the Kubo formula. Indeed, in non-chiral systems this procedure is known to give a quantum derivation of the Boltzmann theory ${ }^{7.8}$ In the case of graphene this program can be fulfilled only retaining the full chiral structure in the explicit calculation of the current vertex corrections. In the limit when the chemical potential is larger than the quasiparticle scattering time an analytical solution can be derived. We show that, in spite of the above argumentations, at least in this regime the results of the Boltzmann theory are still valid, in the sense that the forward-scattering suppression in the transport relaxation rate is still operative, as resulting from the additional angular factor $(1-\cos \theta)$ in $\tau_{\mathrm{tr}}$. This result follows from the fact that the dependence on the momentum angle of the vertex function is strictly replaced by a corresponding angular dependence in the pseudospin space, encoded in the Pauli matrix structure. We investigate the applicability of the Boltzmann results also in the presence of a weak inequivalence between the two carbon sublattices, which, close to the Dirac point, leads to a mixing of the chiral eigenstates $\frac{29,30}{30}$ show that even in this case the Boltzmann results are recovered. The present quantum approach provides in addition a basis for the future calculation of the quantum vertex corrections at finite frequency and in the Dirac limit, which can be both relevant for a direct comparison with existing experimental data. $27,28,31$

The structure of the paper is the following. In Sec. II we introduce the Hilbert space we are working with and the basic formalism for the electron-phonon interaction, while in Sec. III we evaluate the self-energy due to the electron-phonon interaction in graphene. In Sec. IV we implement the calculation of the vertex corrections for the DC conductivity, and we derive the explicit expression for the transport scattering rate. In Sec. V we report the results in the presence of an additional weak inequivalence between the two sublattices, which breaks the chirality preserving the Boltzmann approach. In Sec. VI we discuss some possible observable effects of our analysis in relation to the doping dependence of the high-temperature linear slope of the resistivity, and in Sec. VII we summarize our conclusions. In the Appendices $\mathrm{A}$ and $\mathrm{B}$ we include some details on the calculation of the Eliashberg functions and on the gapped case, respectively.

\section{THE MODEL}

In order to point out the relevance of the angular transport factor $(1-\cos \theta)$ in chiral doped graphene, we consider in this paper the effects of electron scattering with acoustic phonons.

Let us start by introducing the general electron-phonon Hamiltonian in terms of the usual orbital spinor $\psi_{\mathbf{k}}^{\dagger}=$ $\left(c_{\mathbf{k}, \mathrm{A}}^{\dagger}, c_{\mathbf{k}, \mathrm{B}}^{\dagger}\right)$, where $c_{\mathbf{k}, \mathrm{A}}^{\dagger}, c_{\mathbf{k}, \mathrm{B}}^{\dagger}$ represent the creation operator of one electron with momentum $\mathbf{k}$ on the sublattices $\mathrm{A}$ and $\mathrm{B}$ respectively. In this basis we can write:

$$
\begin{aligned}
\hat{H}= & N_{s} \sum_{\mathbf{k}} \psi_{\mathbf{k}}^{\dagger} \hat{H}_{\mathbf{k}}^{0} \psi_{\mathbf{k}}+\sum_{\mathbf{q}, \nu} \omega_{\mathbf{q}, \nu} a_{\mathbf{q}, \nu}^{\dagger} a_{\mathbf{q}, \nu} \\
& +N_{s} \sum_{\mathbf{k}, \mathbf{q}, \nu} \psi_{\mathbf{k}+\mathbf{q}}^{\dagger} \hat{g}_{\mathbf{k}, \mathbf{k}+\mathbf{q}, \nu} \psi_{\mathbf{k}}\left(a_{\mathbf{q}, \nu}+a_{-\mathbf{q}, \nu}^{\dagger}\right) .
\end{aligned}
$$

Here $\hat{H}_{\mathbf{k}}^{0}$ is the non-interacting tight-binding electron Hamiltonian,

$$
\hat{H}_{\mathbf{k}}^{0}=\left(\begin{array}{cc}
0 & f^{*}(\mathbf{k}) \\
f(\mathbf{k}) & 0
\end{array}\right)
$$

where $f^{*}(\mathbf{k})$ is the Fourier-transform of the tight-binding model on the honeycomb lattice, $a_{\mathbf{q}, \nu}^{\dagger}$ is the creation operator of one phonon with momentum $\mathbf{q}$ in the $\nu$ branch, $\omega_{\mathbf{q}, \nu}$ is the corresponding frequency and $\hat{g}_{\mathbf{k}, \mathbf{k}+\mathbf{q}, \nu}$ is the electron-phonon matrix element which presents in general a non trivial matrix structure. $N_{s}=2$ takes into account here the spin degeneracy which will play no role in the following.

In this paper we shall work in the original basis of electron operators for the A and B sublattices, as described in Eq. (11). This choice results to be more convenient in the evaluation of the transport properties from a linear response theory based on the Kubo's formula. As an alternative approach the eigenvector basis, in which the Hamiltonian (11) is diagonal, was employed in Refs ${ }^{20,21,22}$ since it makes easier the implementation of a quantum or quasiclassical extension of the kinetic equations.

A compelling treatment of the electron-phonon interaction including the full phonon spectrum for all q's is a formidable task due to the complex matrix structure of the elements $\hat{g}_{\mathbf{k}, \mathbf{k}+\mathbf{q}, \nu}$. Fortunately, since in weakly doped graphene the electron momenta are close to the Dirac 


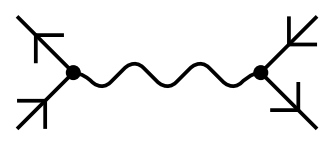

FIG. 1: Diagrammatic representation of the electron-phonon interaction. Straight lines represent incoming and outcoming electrons, the wavy line is the phonon propagator and the filled circles are the el-ph matrix elements.

point, the relevant exchanged phonons are mainly located either close to the $\mathrm{K}, \mathrm{K}^{\prime}$ edge zone or at the $\Gamma(\mathbf{q}=0)$ point. Detailed analysis of the electron-phonon effects for general acoustic and optical modes can be found in Refs. $32,33,34,35,36,37,38,39]$. We concentrate here on the electron-phonon scattering with the acoustic modes with $\mathbf{q} \approx 0$, which are dominant in the $\mathrm{DC}$ transport properties at low temperatures. Several simplifications can be employed in this case. First of all, the phonon dispersion can be simply linearized, $\omega_{\mathbf{q}, \nu} \approx \hbar v_{\mathrm{s}}|\mathbf{q}|$. In addition for $\mathbf{q} \rightarrow 0$ the charge modulation wavelength $\lambda=1 /|\mathbf{q}|$ is much larger than the interatomic distance $a$. In this regime the two carbon atoms of the unitary cell are essentially indistinguishable so that the electron-phonon matrix element behaves as $\hat{g}_{\mathbf{k}, \mathbf{k}+\mathbf{q}} \approx g_{\mathbf{k}, \mathbf{k}+\mathbf{q}} \hat{I}$ in the chiral space. Within the same assumption, we can also neglect intervalley scattering and discuss interactions within a single Dirac cone $\stackrel{24}{\underline{4}}$ Finally, for small doping, we can linearize the electron dispersion close to the Dirac points $\hat{H}_{\mathbf{k}}^{0}=\hbar v_{\mathrm{F}} \mathbf{k} \cdot \boldsymbol{\sigma}$. We can write thus our effective Hamiltonian as:

$$
\begin{aligned}
\hat{H}= & N_{s} N_{k} \sum_{\mathbf{k}} \psi_{\mathbf{k}}^{\dagger} \hbar v_{\mathrm{F}} \mathbf{k} \cdot \boldsymbol{\sigma} \psi_{\mathbf{k}}+\sum_{\mathbf{q}} \hbar v_{\mathrm{s}}|\mathbf{q}| a_{\mathbf{q}}^{\dagger} a_{\mathbf{q}} \\
& +N_{s} N_{K} \sum_{\mathbf{k}, \mathbf{q}} g_{\mathbf{q}} \psi_{\mathbf{k}+\mathbf{q}}^{\dagger} \hat{I} \psi_{\mathbf{k}}\left(a_{\mathbf{q}}+a_{-\mathbf{q}}^{\dagger}\right),
\end{aligned}
$$

where we made use of the relation $g_{\mathbf{k}, \mathbf{k}+\mathbf{q}} \simeq g_{\mathbf{q}}$ for $\mathbf{q} \rightarrow 0$.

The resulting electron-phonon coupling is usually expressed in terms of the kernel $W_{\mathbf{k}-\mathbf{k}^{\prime}}\left(\omega-\omega^{\prime}\right)$, which is diagrammatically depicted in Fig. 1 and which represents the effective retarded interaction between two electrons with momenta $\mathbf{k}, \mathbf{k}^{\prime}$ and energies $\omega, \omega^{\prime}$, which exchange momentum $\mathbf{k}-\mathbf{k}^{\prime}$ and energy $\omega-\omega^{\prime}$. From (2) we have

$$
W_{\mathbf{k}-\mathbf{k}^{\prime}}\left(\omega-\omega^{\prime}\right)=\left|g_{\mathbf{k}-\mathbf{k}^{\prime}}^{2}\right| D_{\mathbf{k}-\mathbf{k}^{\prime}}\left(\omega-\omega^{\prime}\right),
$$

where $D_{\mathbf{q}}(\Omega)=2 \omega_{\mathbf{q}} /\left[\omega_{\mathbf{q}}^{2}-\Omega\right]$ is the phonon propagator. Note that, since $\hat{g}_{\mathbf{q}} \propto \hat{I}$ in the limit $\mathbf{q} \rightarrow 0$, Eq. (3) does not present a matrix structure, simplifying notably the calculations. It is also convenient to express the effective retarded interaction is term of the Eliashberg function $\alpha^{2} F(\mathbf{q}, \Omega)$ :

$$
W_{\mathbf{k}-\mathbf{k}^{\prime}}\left(\omega-\omega^{\prime}\right)=\int d \Omega \frac{2 \Omega \alpha^{2} F\left(\mathbf{k}-\mathbf{k}^{\prime}, \Omega\right)}{\Omega^{2}-\left(\omega-\omega^{\prime}\right)^{2}},
$$

where $\alpha^{2} F\left(\mathbf{k}-\mathbf{k}^{\prime}, \Omega\right)=\left|g_{\mathbf{k}-\mathbf{k}^{\prime}}\right|^{2} \delta\left(\Omega-\omega_{\mathbf{k}-\mathbf{k}^{\prime}}\right)$.

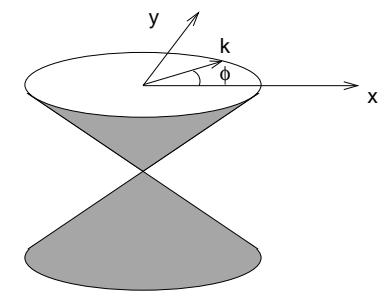

FIG. 2: Sketch of the cylindrical coordinates used in the paper. In this coordinate basis we can write $\mathbf{k}=k(\cos \phi, \sin \phi)$.

In this paper we shall focus on the case of intrinsically doped graphene, where the chemical potential $|\mu|$ is much larger than both the allowed exchanged phonon energies $\omega_{\max }$ and the quasiparticle scattering rate $\Gamma_{\mathrm{qp}}$. Note that in the case of acoustic phonons, the highest exchanged phonon energy $\omega_{\max }$ is given by the BlochGrüneisen energy scale $\varnothing_{\max }=2 \hbar v_{s} k_{\mathrm{F}}$, so that the constraint $|\mu| \gg \omega_{\max }$ implies $v_{\mathrm{F}} \gg v_{s}$, which is always fulfilled in graphene. The condition $\Gamma_{\mathrm{qp}} \ll|\mu|$, on the other hand, is doping-dependent and it is usually fulfilled in doped graphene. In this situation, since electrons are scattered only within a narrow energy window $\pm \omega_{\max }$ around the Fermi level, we can put the electron momenta appearing in the Eliashberg function on the Fermi surface, so that it depends only on the relative angle. Writing $\mathbf{k}=k(\cos \phi, \sin \phi)$ (see Fig. 2), we have thus $k \approx k^{\prime} \approx k_{\mathrm{F}}$, and we can write $|\mathbf{q}|=2 k_{\mathrm{F}} \sin \left[\left(\phi-\phi^{\prime}\right) / 2\right]$ and $\alpha^{2} F\left(\mathbf{k}-\mathbf{k}^{\prime}, \Omega\right)=\alpha^{2} F\left(\phi-\phi^{\prime}, \Omega\right)$. Moreover, the condition $|\mu| \gg \omega_{\max }, \Gamma_{\mathrm{qp}}$ allows us also to restrict ourselves to a single electron (hole) cone for $\mu>0(\mu<0)$, with a significant simplification of the calculations [see Eq. (18) below].

Before discussing the one-particle self-energy, let us briefly summarize the properties of the non interacting system whose Green's function per spin and valley, in the Matsubara space, reads:

$$
\begin{aligned}
\hat{G}^{0}\left(\mathbf{k}, i \omega_{n}\right) & =\frac{1}{\left(i \hbar \omega_{n}+\mu\right) \hat{I}-\hbar v_{\mathrm{F}} \mathbf{k} \cdot \boldsymbol{\sigma}} \\
& =\frac{\left(i \hbar \omega_{n}+\mu\right) \hat{I}+\hbar v_{\mathrm{F}} \mathbf{k} \cdot \boldsymbol{\sigma}}{\left[\left(i \hbar \omega_{n}+\mu\right)\right]^{2}-\left(\hbar v_{\mathrm{F}} k\right)^{2}} .
\end{aligned}
$$

We can expand the Green's function in the Pauli matrix basis,

$$
\hat{G}^{0}\left(\mathbf{k}, i \omega_{n}\right)=\sum_{i=I, x, y} G_{i}^{0}\left(\mathbf{k}, i \omega_{n}\right) \hat{\sigma}_{i} .
$$

It is easy to see, from Eqs. (5)-(6), that the diagonal part $G_{I}^{0}\left(\mathbf{k}, i \omega_{n}\right)$ depends only on the $\epsilon=\hbar v_{\mathrm{F}} k(k=|\mathbf{k}|)$ while the off-diagonal components depend also on the angle $\phi$. In particular, we can write explicitly

$$
\begin{aligned}
& G_{I}^{0}\left(\mathbf{k}, i \omega_{n}\right)=G_{+}^{0}\left(\epsilon, i \omega_{n}\right), \\
& G_{x}^{0}\left(\mathbf{k}, i \omega_{n}\right)=G_{-}^{0}\left(\epsilon, i \omega_{n}\right) \cos \phi, \\
& G_{y}^{0}\left(\mathbf{k}, i \omega_{n}\right)=G_{-}^{0}\left(\epsilon, i \omega_{n}\right) \sin \phi,
\end{aligned}
$$




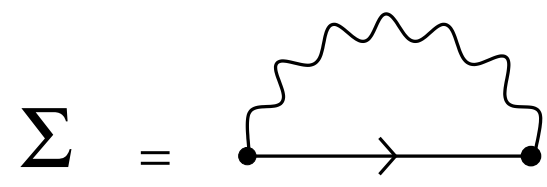

FIG. 3: Diagrammatic representation of the electron-phonon self-energy. Graphical elements as in Fig. 1.

where

$$
G_{ \pm}^{0}\left(\epsilon, i \omega_{n}\right)=\frac{1}{2}\left[\frac{1}{i \hbar \omega_{n}+\mu-\epsilon} \pm \frac{1}{i \hbar \omega_{n}+\mu+\epsilon}\right] .
$$

\section{ONE-PARTICLE SELF-ENERGY}

Let us now consider the one-particle self-energy for the electron-phonon interaction. As mentioned, we are interested here on the limit $|\mu| \gg \omega_{\max }$. In this regime Migdal's theorem $\underline{40}$ assures the validity of the standard mean-field like Eliashberg's theory $\underline{\underline{41}}$ The corresponding self-energy is thus diagrammatically depicted in Fig. 3. and it can be written in Matsubara space as:

$$
\hat{\Sigma}(\mathbf{k}, n)=T \sum_{\mathbf{k}^{\prime}, m} W_{\mathbf{k}-\mathbf{k}^{\prime}}(n-m) \hat{G}\left(\mathbf{k}^{\prime}, m\right) .
$$

where $\hat{G}(\mathbf{k}, n)=\hat{G}\left(\mathbf{k}, i \omega_{n}\right), \hat{\Sigma}(\mathbf{k}, n)=\hat{\Sigma}\left(\mathbf{k}, i \omega_{n}\right)$ and $W_{\mathbf{k}-\mathbf{k}^{\prime}}(n-m)=W_{\mathbf{k}-\mathbf{k}^{\prime}}\left(i \omega_{n}-i \omega_{m}\right)$.

From Dyson's equation we can write:

$$
\hat{G}(\mathbf{k}, n)=\frac{1}{\left(i \hbar \omega_{n}+\mu\right) \hat{I}-\hbar v_{\mathrm{F}} \mathbf{k} \cdot \boldsymbol{\sigma}-\hat{\Sigma}(\mathbf{k}, n)} .
$$

Since Eq. (7) is a convolution both in momentum and frequency space, it is easy to show that the matrix selfenergy admits the analogous decomposition (6) of the Green's function (6), namely

$$
\hat{\Sigma}(\mathbf{k}, n)=\sum_{i=I, x, y} \Sigma_{i}(\mathbf{k}, n) \hat{\sigma}_{i}
$$

where $\Sigma_{I}(\mathbf{k}, n)=\Sigma_{I}(\epsilon, n), \quad \Sigma_{x}(\mathbf{k}, n)=\Sigma_{x}(\epsilon, \phi, n)=$ $\Sigma_{\text {off }}(\epsilon, n) \cos \phi, \Sigma_{y}(\mathbf{k}, n)=\Sigma_{y}(\epsilon, \phi, n)=\Sigma_{\text {off }}(\epsilon, n) \sin \phi$, where the label "off' characterizes the off-diagonal elements of the self-energy.

Using the Dyson's equation, we can write once more $G_{I}(\mathbf{k}, n)=G_{+}(k, n), G_{x}(\mathbf{k}, n)=G_{-}(\epsilon, n) \cos \phi$, $G_{y}(\mathbf{k}, n)=G_{-}(\epsilon, n) \sin \phi$, with

$$
\begin{aligned}
G_{ \pm}(\epsilon, n)= & \frac{1}{2}\left[\frac{1}{i \hbar \omega_{n}+\mu-\epsilon-\Sigma_{I}(\epsilon, n)-\Sigma_{\text {off }}(\epsilon, n)}\right. \\
& \left. \pm \frac{1}{i \hbar \omega_{n}+\mu+\epsilon-\Sigma_{I}(\epsilon, n)+\Sigma_{\text {off }}(\epsilon, n)}\right](.8)
\end{aligned}
$$

The calculation of Eq. (7) is straightforward in the case of intrinsically doped graphene. As discussed above, the relevant electron momenta are here restricted on the Fermi surface, $\mathbf{k} \approx k_{\mathrm{F}}(\epsilon=\mu)$, so that the self-energy (77) depends only on the angular part of the $\mathbf{k}$ vector, $\Sigma_{i}(\epsilon, \phi, n) \approx \Sigma_{i}(\phi, n)$. We can also split the sum over $\mathbf{k}^{\prime}$ in Eq. (7) in its energy and angular degrees of freedom, $\sum_{\mathbf{k}} \longrightarrow \int_{-\pi}^{\pi} d \phi / 2 \pi \int_{0}^{W} d \epsilon N(\epsilon)$, where $W$ is the electron bandwidth, and $N(\epsilon)=\epsilon V / 2 \pi \hbar^{2} v_{\mathrm{F}}^{2}$ is the density of states per spin and per valley, and $\mathrm{V}$ is the unit-cell volume. Thus we have

$$
\Sigma_{i}(\phi, n)=T \sum_{m} \int \frac{d \phi^{\prime}}{2 \pi} W_{\phi-\phi^{\prime}}(n-m) \chi_{i}\left(\phi^{\prime}\right) G_{i, \mathrm{loc}}(m),
$$

where $\chi_{I}=1, \chi_{x}(\phi)=\cos \phi, \chi_{y}(\phi)=\sin \phi$, and $G_{i, \text { loc }}(m)$ is the local (k-averaged) Green's function: $\quad G_{I, \text { loc }}(m)=G_{+, \text {loc }}(m)=\int N(\epsilon) d \epsilon G_{+}(\epsilon, m)$, $G_{x, y, \text { loc }}(m)=G_{-, \text {loc }}(m)=\int N(\epsilon) d \epsilon G_{-}(\epsilon, m)$.

It is useful at this stage to introduce the basis of the two-dimensional spherical harmonics

$$
\psi_{\alpha}(\phi)=e^{i \alpha \phi}, \quad \alpha=0, \pm 1, \pm 2, \ldots,
$$

so that we can decompose any generic angle-dependent function $S(\phi)$ on this basis,

$$
S(\phi)=\sum_{\alpha} S_{\alpha} \psi_{\alpha}(\phi)
$$

where

$$
S_{\alpha}=\int \frac{d \theta}{2 \pi} S(\phi) \psi_{\alpha}^{*}(\phi) .
$$

Using the definition (4) of the electron-phonon kernel, we can write the diagonal and off-diagonal components of the self-energy in Eq. (9) as

$$
\begin{aligned}
\Sigma_{I}(n) & =T \sum_{m} \int d \Omega \frac{2 \Omega \alpha^{2} F_{0}(\Omega)}{\Omega^{2}+\left(\omega_{n}-\omega_{m}\right)^{2}} G_{+, \mathrm{loc}}(m) \\
\Sigma_{\mathrm{off}}(n) & =T \sum_{m} \int d \Omega \frac{2 \Omega \alpha^{2} F_{1}(\Omega)}{\Omega^{2}+\left(\omega_{n}-\omega_{m}\right)^{2}} G_{-, \mathrm{loc}}(m)
\end{aligned}
$$

where $\alpha^{2} F_{\alpha}$ are the projections of the Eliashberg function $\alpha^{2} F(\phi)$ on the spherical harmonics $\psi_{\alpha}(\phi)$, according to the decomposition (10) above.

Eqs. (12)-(13) can be easily analytically continued on the real-frequency axis using standard techniques. Through the imaginary part of the self-energy, we can thus define a diagonal and an off-diagonal scattering rate, $\Gamma_{I(\text { off })}=-\lim _{\omega \rightarrow 0} \operatorname{Im} \Sigma_{I(\text { off })}\left(\omega+i 0^{+}\right)$, where

$$
\begin{aligned}
\Gamma_{I}= & -2 \pi N(\mu) \int d \Omega \alpha^{2} F_{0}(\Omega)[n(\beta \Omega)+f(\beta \Omega)] \\
& \times \operatorname{Im}\left[G_{+, \operatorname{loc}}\left(\Omega+i 0^{+}\right)\right],
\end{aligned}
$$

and where

$$
\begin{aligned}
\Gamma_{\text {off }}= & -2 \pi N(\mu) \int d \Omega \alpha^{2} F_{1}(\Omega)[n(\beta \Omega)+f(\beta \Omega)] \\
& \times \operatorname{Im}\left[G_{-, \text {loc }}\left(\Omega+i 0^{+}\right)\right] .
\end{aligned}
$$


Here $n(x)=1 /\left[\mathrm{e}^{x}-1\right]$ and $f(x)=1 /\left[\mathrm{e}^{x}+1\right]$ are the Bose and Fermi factors, respectively.

For a practical evaluation of the self-energy terms, we can make use once more of the fact that, due to the lowenergy phonon-mediated scattering, the relevant electron energy are restricted to the Fermi level. In this case we can write $\int_{0}^{W} d \epsilon N(\epsilon) \approx N(\mu) \int_{0}^{W} d \epsilon$ and, assuming $\mu>0$, it is easy to see that only the upper Dirac cone [first term in Eq. (8)] is relevant. We have in particular $G_{+, \text {loc }}\left(\Omega+i 0^{+}\right) \approx G_{-, \text {loc }}\left(\Omega+i 0^{+}\right)=-i \pi N(\mu) / 2$, so that, just as in common metals, the real-part of the self-energy vanishes, and we have $\Gamma_{\mathrm{I}}=2 K_{0}, \Gamma_{\text {off }}=2 K_{1}$, where

$$
K_{\alpha}=\frac{\pi N(\mu)}{2} \int d \varnothing \alpha^{2} F_{\alpha}(\varnothing)[n(\beta \varnothing)+f(\beta \varnothing)] .
$$

For the states at the Fermi energy, which involve only the upper band $(\mu>0)$, we can define thus a total quasiparticle scattering rate as

$$
\Gamma_{\mathrm{qp}}=\Gamma_{\mathrm{I}}+\Gamma_{\mathrm{off}}=2 K_{0}+2 K_{1},
$$

which, using Eq. (8), gives the dressed Green's functions:

$$
G_{+}(\epsilon, \omega) \approx G_{-}(\epsilon, \omega) \approx \frac{1}{2} \frac{1}{\hbar \omega+\mu-\epsilon+i \Gamma_{\mathrm{qp}}} .
$$

From Eq. (14)-(17) we obtain finally:

$$
\begin{aligned}
\Gamma_{\mathrm{qp}} & =\pi N(\mu) \sum_{i=0,1} \int d \Omega \alpha^{2} F_{i}(\Omega)[n(\beta \Omega)+f(\beta \Omega)], \\
& =\pi N(\mu) \int \frac{d \theta}{2 \pi} g_{\theta}^{2}(1+\cos \theta)\left[n\left(\beta \emptyset_{\theta}\right)+f\left(\beta \emptyset_{\theta}\right)\right],
\end{aligned}
$$

where $g_{\theta}^{2}=I|\mathbf{q}|=I 2 k_{F} \sin (\theta / 2)$ and $\varnothing_{\theta}=\hbar v_{s}|\mathbf{q}|=$ $2 \hbar v_{s} k_{\mathrm{F}} \sin (\theta / 2)$. Using the explicit expression for the $K_{\alpha}$ coefficients (see Appendix $\mathrm{A}$ ), we obtain standard results with a $\Gamma_{\mathrm{qp}}=\left(\pi^{2} N(\mu) I / \hbar \omega_{\max } v_{s}\right) T^{2}$ in the BlochGrüneisen regime $\left(T \lesssim \varnothing_{\max }\right)$ and $\Gamma_{\mathrm{qp}}=\left(\pi N(\mu) I / \hbar v_{s}\right) T$ in the high-temperature $T \gtrsim \emptyset_{\max }$ regime.

It is important to note that the total quasi-particle scattering rate arises from both the diagonal and offdiagonal contributions of the self-energy. Indeed, the explicit angular dependence shown in the second line of Eq. (19) points out that the off-diagonal terms, giving rise to the $\cos \theta$, are fundamental in order to recover the usual $(1+\cos \theta)$ factor, which accounts for the well-known absence of backscattering in graphene due to chirality: $\underline{14,18,19}$

\section{DC CONDUCTIVITY}

We implement now a full quantum treatment to evaluate the DC conductivity $\sigma$ in the presence of vertex

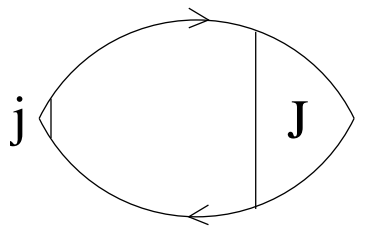

FIG. 4: Diagrammatic representation of the the currentcurrent response function $\Pi$. The small empty triangle on the left represents the bare current vertex $\hat{j}(\mathbf{k})$ while the big triangle on the right is the renormalized current vertex function $\hat{J}(\mathbf{k} ; n, n+m)$.

current renormalization. We consider thus the currentcurrent response function $\stackrel{7,8}{*}$

$$
\begin{aligned}
\Pi(m)= & \frac{N_{s} N_{K}}{V} T \sum_{\mathbf{k}, n} \operatorname{Tr}[\hat{j}(\mathbf{k}) \hat{G}(\mathbf{k}, n) \\
& \times \hat{J}(\mathbf{k} ; n, n+m) \hat{G}(\mathbf{k}, n+m)],
\end{aligned}
$$

where $N_{s}, N_{K}=2$ are respectively the spin and valley degeneracies, $j(\mathbf{k})=e v_{\mathrm{F}} \hat{\sigma}_{x}$ is the bare current operator along the $x$-axis, and $\hat{J}(\mathbf{k} ; n, n+m)$ is the fully renormalized vertex current. The diagrammatic representation of the current-current response function is shown in Fig. 4. The DC conductivity will be obtained as the limit $\sigma=-\lim _{\omega \rightarrow 0} \operatorname{Im} \Pi\left(\omega+i 0^{+}\right) / \omega$ after the analytical continuation of the current-current response function $\Pi$ on the real frequency axis.

It is useful to remark here the importance in transport properties of the current renormalization processes which account for the backflow cloud associated to the current of the quasiparticles. As discussed in the introduction, one of the main effects of such current renormalization in normal metals is to give rise to the angular factor $(1-\cos \theta)$ which differentiates the transport scattering rate $\Gamma_{\mathrm{tr}}$ from the quasi-particle one $\Gamma_{\mathrm{qp}}$. We can understand this result by noting that, in normal (isotropic) metals, the bare and the renormalized currents, $\mathbf{j}$ and $\mathbf{J}$ respectively, are both proportional to the velocity, i.e. they point in the direction of the momentum k. Thus, one can write $\mathbf{J}=\Lambda \mathbf{k}$, where the vertex function $\mathrm{E}$ can be computed for instance in the ladder approximation. The self-consistent solution gives thus: ${ }^{\underline{8}}$

$$
\left\langle\Gamma_{\mathrm{tr}}\right\rangle_{\theta} \simeq\left\langle\Gamma_{\mathrm{qp}}\left(1-\frac{\mathbf{k} \cdot \mathbf{k}^{\prime}}{k^{2}}\right)\right\rangle_{\theta},
$$

where $\mathbf{k}, \mathbf{k}^{\prime}$ are the momenta of the scattered electrons, with modulus equal to $k_{\mathrm{F}}$ but different directions, and $\langle\ldots\rangle_{\theta}$ indicates the angular integration. This leads to the usual additional factor $\left(1-\mathbf{k} \cdot \mathbf{k}^{\prime} / k^{2}\right)=(1-\cos \theta)$ in the angular average of the transport scattering time, which reproduces by means of a full quantum treatment the well-known semiclassical Boltzmann result.

While deriving the result (21), a crucial ingredient is the proportionality between $\mathbf{J}$ and the momentum $\mathbf{k}$. In 
graphene, where the energy-momentum dispersion is linear, such a relation clearly does not hold. However, as it is evident already at the level of the bare current $\hat{j}$, the matrix structure plays the analogous role of the momentum dependence in ordinary metals, so that $\hat{j}_{x}$ for example is a matrix proportional to $\hat{\sigma}_{x}$. As far as the renormalized current is concerned, the analogous of the momentum dependence in the ordinary metals becomes now a decomposition of $\hat{J}$ in the Pauli-matrices components, by means of dimensionless function $\hat{\Lambda}$ defined by the relation $\hat{J}(\mathbf{k} ; n, n+m)=e v_{\mathrm{F}} \hat{\Lambda}(\mathbf{k} ; n, n+m)$. As we shall see in what follows, in graphene such a matrix structure compensates - in a non-trivial way - the lack of momentum dependence of the Fermi velocity, and leads once more to the Boltzmann result.

Aiming on focusing on the matricial structure of the current function, in the following we shall make use for this quantities of the same approximations employed for the self-energy. In particular, taking into account that the electron-phonon interaction gives rise only to lowenergy scattering, we can approximate $\mathbf{k} \simeq k_{\mathrm{F}}$ in the vertex function and retain only the angular dependence. In addition, one can decompose the vertex function in the basis of the Pauli-matrices,

$$
\hat{\Lambda}(\phi ; n, n+m)=\sum_{i} \Lambda_{i}(\phi ; n, n+m) \hat{\sigma}_{i},
$$

and we can expand the $\Lambda_{i}(\phi)$ functions in terms of the spherical harmonics components as in Eq. (10): $\Lambda_{i}(\phi ; n, n+m)=\sum_{\alpha} \Lambda_{i}^{\alpha}(n, n+m) \psi_{\alpha}(\phi)$. Inserting Eq. (22) in Eq. (20), and assuming once more, for doped graphene, $G_{+} \approx G_{-}$(neglect of inter-band scattering), we obtain thus the general structure:

$$
\Pi(m)=\frac{2 e^{2} v_{\mathrm{F}}^{2} N_{K} N_{S}}{V} T \sum_{n} \mathrm{E}^{\mathrm{tot}}(n, n+m) b(n, n+m),
$$

where $b(n, n+m)=N(\mu) \int d \epsilon G(\epsilon, n) G(\epsilon, n+m)$ (since $G_{+}=G_{-}$we have further dropped the index " \pm ") and where $\mathrm{E}^{\text {tot }}(n, n+m)=\sum_{i, \alpha} c_{i}^{\alpha} \mathrm{E}_{i}^{\alpha}(n, n+m)$. The $c_{i}^{\alpha}$ are numerical coefficients which arise from the angular average over $\phi$. Since in the bubble (20) one has the product of two Green's functions, it is easy to realize that the angular average will involve at most harmonics up to the second order. Indeed, an explicit calculation shows that the only non vanishing terms are

$$
\begin{aligned}
c_{x}^{0} & =c_{I}^{1}=c_{I}^{-1}=1, \\
c_{x}^{2} & =c_{x}^{-2}=-i c_{y}^{2}=i c_{y}^{-2}=\frac{1}{2} .
\end{aligned}
$$

Once the analytical continuation $i \omega_{m} \rightarrow \omega+i 0^{+}$of the bubble (23) is performed and the limit $\omega \rightarrow 0$ is evaluated to compute the DC conductivity, one can see ${ }^{8}$ that only the advanced-retarded parts of the vertex function and of the $b$-function contribute, so that:

$$
\sigma \simeq \frac{N_{K} N_{s} \hbar e^{2} v_{\mathrm{F}}^{2}}{\pi V} b_{\mathrm{RA}}(0) \mathrm{E}_{\mathrm{RA}}^{\mathrm{tot}}(0)
$$

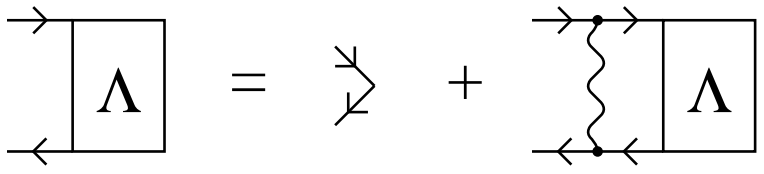

FIG. 5: Diagrammatic representation of the current vertex function. Graphical elements as in Fig. 11 The ladder approximation is enforced by the Migdal's theorem valid for $|\mu| \gg \omega_{\max }$

where $b_{\mathrm{RA}}(\epsilon)=\lim _{\omega \rightarrow 0} b\left(\omega+i 0^{+}, \omega-i 0^{+}\right)$, and $\mathrm{七}_{\mathrm{RA}}^{\mathrm{tot}}(0)=$ $\lim _{\omega \rightarrow 0} \mathrm{E}^{\text {tot }}\left(\omega+i 0^{+}, \omega-i 0^{+}\right)$. From Eq. (18) one easily finds

$$
b_{\mathrm{RA}}(0)=\frac{N(\mu) \pi}{4 \Gamma_{\mathrm{qp}}}
$$

which gives the general expression for the DC conductivity in the presence of vertex corrections:

$$
\sigma=\frac{N_{K} N_{s} \hbar e^{2} v_{\mathrm{F}}^{2} N(\mu)}{4 V \Gamma_{\mathrm{tr}}}
$$

where we define the transport scattering rate

$$
\Gamma_{\mathrm{tr}}=\frac{\Gamma_{\mathrm{qp}}}{\mathrm{E}_{\mathrm{RA}}^{\mathrm{tot}}(0)}
$$

In the absence of vertex corrections $\left(\mathrm{E}_{\mathrm{RA}}^{\mathrm{tot}}(0)=1\right)$ one recognizes in Eq. (27), as mentioned above in the case of ordinary metals, the standard result of the conductivity in the bare-bubble approximation, $, 24,42$ where the scattering time for transport coincides with the quasi-particle scattering time $\sigma=e^{2} \mu /\left(2 \pi \hbar \Gamma_{\mathrm{qp}}\right)$.

To compute Eq. (26) we need then to calculate the vertex corrections. In the doped graphene regime we are interested in, this aim is made easy once more by the Migdal's theorem which enforces the validity of the mean-field theory. The corresponding vertex function $\hat{\Lambda}(\mathbf{k} ; n, n+m)$ can be evaluated thus within the selfconsistent ladder approximation (see Fig. 5), namely: ${ }^{8}$

$$
\begin{aligned}
& \hat{\Lambda}(\mathbf{k} ; n, n+m)=\hat{\sigma}_{x}+T \sum_{\mathbf{k}^{\prime}, l} W_{\mathbf{k}-\mathbf{k}^{\prime}}(n-l) \\
& \times \hat{G}\left(\mathbf{k}^{\prime}, l\right) \hat{\Lambda}\left(\mathbf{k}^{\prime} ; l, l+m\right) \hat{G}\left(\mathbf{k}^{\prime}, l+m\right) .
\end{aligned}
$$

In the same limit $|\mu| \gg \omega_{\max }$, we can also employ the approximations implemented for the self-energy, namely $|\mathbf{k}| \approx\left|\mathbf{k}^{\prime}\right| \approx k_{\mathrm{F}}, \sum_{\mathbf{k}^{\prime}} \longrightarrow N(\mu) \int_{-\pi}^{\pi} d \phi^{\prime} / 2 \pi \int_{-\infty}^{\infty} d \epsilon^{\prime}$, and we can also set $\hat{\Lambda}\left(\mathbf{k}_{\mathrm{F}}^{\prime} ; l, l+m\right)=\hat{\Lambda}\left(\phi^{\prime} ; l, l+m\right)$ in the righthand side of Eq. (29). Expanding again the $\hat{\Lambda}\left(\phi^{\prime} ; l, l+m\right)$ in terms of the Pauli basis and of the spherical harmonics, 
we end up with the following set of equations

$$
\begin{aligned}
\Lambda_{x}^{\alpha}(n, n+m)= & \delta_{\alpha, 0}+T \sum_{l, j, \beta} c_{j}^{\beta} W_{\alpha}(n-l) \\
& \times b(l, l+m) \Lambda_{j}^{\beta+\alpha}(l, l+m), \\
\Lambda_{I}^{\alpha}(n, n+m)= & T \sum_{l, j, \beta} d_{j}^{\beta} W_{\alpha}(n-l) \\
& \times b(l, l+m) \Lambda_{j}^{\beta+\alpha}(l, l+m), \\
\Lambda_{y}^{\alpha}(n, n+m)= & T \sum_{l, j, \beta} f_{j}^{\beta} W_{\alpha}(n-l) \\
& \times b(l, l+m) \Lambda_{j}^{\beta+\alpha}(l, l+m),
\end{aligned}
$$

where the coefficients $c_{i}^{\alpha}$ are defined in Eqs. (24)-(25), and the only non zero terms of the coefficients $d_{i}^{\alpha}, f_{i}^{\alpha}$ are:

$$
\begin{aligned}
d_{I}^{0} & =2 \\
d_{x}^{1} & =d_{x}^{-1}=-i d_{y}^{1}=i d_{y}^{-1}=1, \\
f_{y}^{0} & =-i f_{I}^{1}=i f_{I}^{-1}=1 \\
-i f_{x}^{2} & =i f_{x}^{-2}=-f_{y}^{2}=-f_{y}^{-2}=\frac{1}{2} .
\end{aligned}
$$

We consider now the analytical continuation of Eqs. (30)-(32) on the real axis. We are interested on the quantity $\Lambda_{i, \mathrm{RA}}^{\alpha}(0)$. We can apply the usual standard procedures for the analytical continuation ${ }^{8}$ to each element on the right side of Eqs. (30)-(32), and we get, for instance,

$$
\begin{aligned}
\Lambda_{x, \mathrm{RA}}^{\alpha}(0)= & \delta_{\alpha, 0}+\frac{\pi N(\mu)}{2} \int d \Omega \alpha^{2} F_{\alpha}(\Omega) \\
& \times[n(\beta \Omega)+f(\beta \Omega)]\left[\sum_{j, \beta} c_{j}^{\beta} \frac{\Lambda_{j, \mathrm{RA}}^{\beta+\alpha}(\Omega)}{\Gamma_{\mathrm{qp}}(\Omega)}\right](33)
\end{aligned}
$$

Similar expressions hold true for $\Lambda_{I, \mathrm{RA}}^{\alpha}(0), \Lambda_{y, \mathrm{RA}}^{\alpha}(0)$.

The quantities $\Lambda_{j, \mathrm{RA}}^{\beta}(\Omega), \Gamma_{\mathrm{qp}}(\Omega)$ in the right side of Eq. (33) have a significant $\Omega$ variation over an electronic range of energies, whereas the Eliashberg function $\alpha^{2} F_{\alpha}(\Omega)$ limits the energy integration up to the phonon scale $\omega_{\max }$. In this energy range we can then approximate in the integral of Eq. (33),$\Lambda_{j, \mathrm{RA}}^{\beta}(\Omega) \simeq \Lambda_{j, \mathrm{RA}}^{\beta}(0)$, $\Gamma_{\mathrm{qp}}(\Omega) \simeq \Gamma_{\mathrm{qp}}(0)=\Gamma_{\mathrm{qp}}$, and we obtain a simple set of $a l$ gebraic relations for the vertex function at zero frequency,

$$
\begin{aligned}
\Lambda_{x, \mathrm{RA}}^{\alpha}(0) & =\delta_{\alpha, 0}+\frac{K_{\alpha}}{\Gamma_{\mathrm{qp}}} \sum_{j, \beta} c_{j}^{\beta} \Lambda_{j, \mathrm{RA}}^{\beta+\alpha}(0), \\
\Lambda_{I, \mathrm{RA}}^{\alpha}(0) & =\frac{K_{\alpha}}{\Gamma_{\mathrm{qp}}} \sum_{j, \beta} d_{j}^{\beta} \Lambda_{j, \mathrm{RA}}^{\beta+\alpha}(0), \\
\Lambda_{y, \mathrm{RA}}^{\alpha}(0) & =\frac{K_{\alpha}}{\Gamma_{\mathrm{qp}}} \sum_{j, \beta} f_{j}^{\beta} \Lambda_{j, \mathrm{RA}}^{\beta+\alpha}(0) .
\end{aligned}
$$

Despite its apparent complexity, such a system of equations admits a simple solution. In particular, by exploiting the symmetric/antisymmetric properties for $\alpha \rightarrow-\alpha$ of the $x, I$ and $y$ components, respectively, one can see that only three independent components are not zero, i.e. $\Lambda_{x, \mathrm{RA}}^{0}, \Lambda_{x, \mathrm{RA}}^{2}, \Lambda_{I, \mathrm{RA}}^{1}$, with the relations, $\Lambda_{x, \mathrm{RA}}^{2}=\Lambda_{x, \mathrm{RA}}^{-2}=i \Lambda_{y, \mathrm{RA}}^{2}=-i \Lambda_{y, \mathrm{RA}}^{-2}, \Lambda_{I, \mathrm{RA}}^{1}=\Lambda_{I, \mathrm{RA}}^{-1}$. The system can be further simplified by noting that it can be rewritten in terms of a single self-consistent equation for $\Lambda_{\mathrm{RA}}^{\text {tot }}$ :

$$
\Lambda_{\mathrm{RA}}^{\mathrm{tot}}=1+\frac{K_{0}+2 K_{1}+K_{2}}{\Gamma_{\mathrm{qp}}} \Lambda_{\mathrm{RA}}^{\mathrm{tot}},
$$

whose solution gives

$$
\Lambda_{\mathrm{RA}}^{\text {tot }}(0)=\frac{\Gamma_{\mathrm{qp}}}{\Gamma_{\mathrm{qp}}-K_{0}-2 K_{1}-K_{2}} .
$$

Finally, by expressing the quasi-particle scattering rate $\Gamma_{\text {qp }}$ as a function of the $K_{\alpha}$ terms as in Eq. (17) we obtain:

$$
\Gamma_{\mathrm{tr}}=K_{0}-K_{2}
$$

Eqs. (34), 35) are the main result of the present paper. Their physical insight appears clearly if we express the transport scattering rate in terms of the microscopic electron-phonon interaction, in analogy with the result (19) for the quasiparticle scattering rate:

$$
\begin{aligned}
\Gamma_{\mathrm{tr}}= & K_{0}-K_{2} \\
= & \pi N(\mu) \int \frac{d \theta}{2 \pi} g_{\theta}^{2} \frac{1-\cos 2 \theta}{2}\left[n\left(\beta \emptyset_{\theta}\right)+f\left(\beta \emptyset_{\theta}\right)\right] \\
= & \pi N(\mu) \int \frac{d \theta}{2 \pi} g_{\theta}^{2}(1-\cos \theta)(1+\cos \theta) \\
& \times\left[n\left(\beta \emptyset_{\theta}\right)+f\left(\beta \emptyset_{\theta}\right)\right] .
\end{aligned}
$$

Taking into account the expression of the quasi-particle scattering rate $\Gamma_{\mathrm{qp}}$ in Eq. (19), we can write Eq. (36) as

$$
\left\langle\Gamma_{\mathrm{tr}}\right\rangle_{\theta}=\left\langle\Gamma_{\mathrm{qp}}(1-\cos \theta)\right\rangle_{\theta}
$$

This analysis shows thus that a fully quantum derivation of the DC conductivity yields in doped graphene the same result than the standard Boltzmann theory. In particular we can see that, although the vertex function is $\mathbf{k}$-independent in graphene, the effect of current vertex corrections is to add, just as in common systems, the additional angular factor $1-\cos \theta$ in the phase space probed by the electron-phonon interaction, giving rise to a suppression of the forward scattering. The effects of such angular factor can be remarkably traced in the temperature dependence of the transport scattering rate $\Gamma_{\mathrm{tr}}=K_{0}-K_{2}$ (and hence of the resistivity $\left.\rho=1 / \sigma\right)$ compared with the quasi-particle one $\Gamma_{\mathrm{qp}}=2\left(K_{0}+K_{1}\right)$. An analytical derivation of the $K_{\alpha}$ coefficients is reported in Appendix $\mathrm{A}$ and the corresponding temperature dependence of $\Gamma_{\mathrm{tr}}$ and $\Gamma_{\mathrm{qp}}$ is shown in Fig. 6. These results are pretty well consistent with the standard Boltzmann theory $\stackrel{18,19}{ }$ In the high temperature limit $T \gg \varnothing_{\max }$ only 


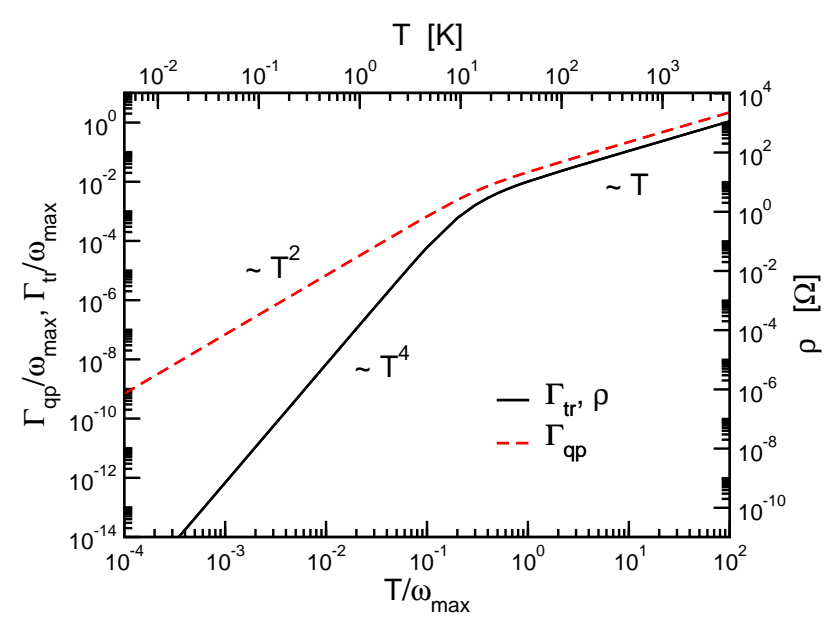

FIG. 6: (Color online). Temperature behavior of the quasiparticle scattering rate $\Gamma_{\mathrm{qp}}$ and of the transport scattering rate $\Gamma_{\operatorname{tr}}$ for $\mu=0.1 \mathrm{eV}$, corresponding to $\emptyset_{\max } \approx 4 \mathrm{meV}$. The transport scattering rate $\Gamma_{\operatorname{tr}}$ can be also expressed as a function of resistivity, $\rho=4 V \Gamma_{\mathrm{tr}} / N_{K} N_{s} \hbar e^{2} v_{\mathrm{F}}^{2} N(\mu)$, as plotted on the right-side scale.

the $K_{0}$ component survives, with $K_{0}=\pi N(\mu) I T / 2 \hbar v_{s}$ (see Appendix $\left(\mathrm{A}\right.$ ), so that $\Gamma_{\mathrm{tr}}, \Gamma_{\mathrm{qp}} \sim T$. On the other hand in the Bloch-Grüneisen regime, just as in common metals, we recover the usual $\Gamma_{\mathrm{tr}} \sim T^{4}$ while $\Gamma_{\mathrm{qp}} \sim T^{2}$. This different dependence can be understood by noting that the low temperature behavior of $\Gamma_{\mathrm{qp}}$ in Eq. (17) is dominated by the leading order $\sim T^{2}$ of $K_{0}, K_{1}$, whereas the leading orders $K_{0}, K_{2}$ in Eq. (35) cancel out so that the temperature behavior of $\Gamma_{\mathrm{tr}}$ in this regime stems from the higher order $\sim T^{4}$ contributions.

\section{BREAKING THE CHIRALITY}

In the previous Section we have evaluate the DC conductivity in chiral doped graphene by using a quantum approach based on the Kubo formula. We have shown that, although the vertex function is momentum independent, the Boltzmann results are fully reproduced, even for what concerns the presence of the so-called angular transport factor $1-\cos \theta$ which suppresses forward scattering. We would like to stress however that the resulting validity of the Boltzmann theory is by no means trivial. Indeed, as we have shown, the robustness of the Boltzmann results is due to the chiral structure itself of graphene, $\hat{H}_{\mathbf{k}}=\hbar v_{\mathrm{F}} \mathbf{k} \cdot \boldsymbol{\sigma}$, which translate in the direction of the Pauli matrix pseudospin $\sigma$ the role which in common metals is played by the momentum k-direction.

Such observation rises the question about the validity of the Boltzmann result when the chiral structure of graphene is affected by external fields. This happens for instance in the presence of a weak sublattice inequivalence, where an energy potential difference between the two sublattices, besides opening a gap at the Dirac point,$\stackrel{34,43}{\longleftarrow}$ gives rise also to a mixing of the chiral eigenstates and to a loss of chirality close to the Dirac point. Such reduced role of the chirality close to the Dirac point has been traced, for instance, in Ref. 29, 30 in the intensity profile along the constant-energy contours probed by angle-resolved photoemission $\stackrel{29.30}{=}$ The validity of the Boltzmann results even in this context needs thus to be revised.

To this aim in this section we consider doped graphene in the presence of a weak inequivalence between the two carbon sublattices, parametrized in terms of a different sublattice potential $\Delta$ :

$$
\hat{H}_{\mathbf{k}}=\hbar v_{\mathrm{F}} \mathbf{k} \cdot \boldsymbol{\sigma}+\Delta \hat{\sigma}_{z} .
$$

The energy spectrum is easily obtained from (38), $E_{k}=$ $\sqrt{\left(\hbar v_{\mathrm{F}} k\right)^{2}+\Delta^{2}}$, showing the opening of a gap at the Dirac point.

Let us consider for the moment the case of a noninteracting system. The presence of $\Delta$ induces an explicit $\propto \hat{\sigma}_{z}$ component in both the Green's function and self-energy. Eq. (6) is thus generalized as $\hat{G}(\mathbf{k}, n)=\sum_{i=I, x, y, z} G_{i}(\mathbf{k}, n) \hat{\sigma}_{i}$, where $G_{I}(\mathbf{k}, n)=$ $G_{+}(E, n), G_{x}(\mathbf{k}, n)=\gamma_{\text {off }}(E) G_{-}(E, n) \cos \phi, G_{y}(\mathbf{k}, n)=$ $\gamma_{\text {off }}(E) G_{-}(E, n) \sin \phi, G_{z}(\mathbf{k}, n)=\gamma_{z}(E) G_{-}(E, n)$, with

$$
G_{ \pm}(E, n)=\frac{1}{2}\left[\frac{1}{i \hbar \omega_{n}+\mu-E} \pm \frac{1}{i \hbar \omega_{n}+\mu+E}\right],
$$

$\gamma_{\text {off }}(E)=\epsilon / E, \gamma_{z}(E)=\Delta / E$. Here we denote $\epsilon=\hbar v_{\mathrm{F}} k$ and $E=\sqrt{\epsilon^{2}+\Delta^{2}}$.

Since we are interested in the doped graphene, where $|\mu| \gg \omega_{\max }$, and we assume $\mu>0$, we can as usual consider only the upper band, and we can restrict the electronic states on the Fermi surface, for which $E \approx \mu$, $\epsilon \approx \sqrt{1-\Delta^{2} / \mu^{2}}$, and we have

$$
G_{i}(E, n)=\frac{\gamma_{i}}{2} g(E, n),
$$

where $\gamma_{I}=1, \gamma_{x}=\gamma_{y}=\gamma_{\text {off }}=\sqrt{1-\Delta^{2} / \mu^{2}}, \gamma_{z}=\Delta / \mu$ and where $g(E, n)=1 /\left(i \hbar \omega_{n}+\mu-E\right)$.

Plugging Eq. (39) in (7), we can write

$$
\begin{aligned}
\Sigma_{I}(n) & =\frac{T}{2} \sum_{m} W(n-m) g_{\mathrm{loc}}(m), \\
\Sigma_{\mathrm{off}}(n) & =\frac{\gamma_{\mathrm{off}} T}{2} \sum_{m} W_{1}(n-m) g_{\mathrm{loc}}(m), \\
\Sigma_{z}(n) & =\frac{\gamma_{z} T}{2} \sum_{m} W(n-m) g_{\mathrm{loc}}(m),
\end{aligned}
$$

where $g_{\text {loc }}(m)=N(\mu) \int d E g(E, m)$. After the standard analytical continuation on the real-axis, we can see as usual that the real part of self-energy vanishes and the imaginary parts of the different components give rise to the corresponding scattering rates

$$
\begin{aligned}
\Gamma_{I} & =2 K_{0}, \\
\Gamma_{\mathrm{off}} & =\gamma_{\mathrm{off}} 2 K_{1}, \\
\Gamma_{z} & =\gamma_{z} 2 K_{0} .
\end{aligned}
$$


Inserting this relations in the matrix expression for the Green's function, after some careful derivation described in Appendix B, we can write a Green's function in the presence of interaction in the form of Eq. (18), with

$$
\begin{aligned}
\Gamma_{\mathrm{qp}} & =\Gamma_{I}+\gamma_{\mathrm{off}} \Gamma_{\mathrm{off}}+\gamma_{z} \Gamma_{z} \\
& =\left(1+\gamma_{z}^{2}\right) 2 K_{0}+\gamma_{\mathrm{off}}^{2} 2 K_{1} \\
& =\left(1+\frac{\Delta^{2}}{\mu^{2}}\right) 2 K_{0}+\left(1-\frac{\Delta^{2}}{\mu^{2}}\right) 2 K_{1}
\end{aligned}
$$

We can write thus

$$
\begin{aligned}
\Gamma_{\mathrm{qp}}= & \pi N(\mu) \int \frac{d \theta}{2 \pi} g_{\theta}^{2}\left[n\left(\beta \emptyset_{\theta}\right)+f\left(\beta \varnothing_{\theta}\right)\right] \\
& \times\left[\left(1+\frac{\Delta^{2}}{\mu^{2}}\right)+\left(1-\frac{\Delta^{2}}{\mu^{2}}\right) \cos \theta\right] .
\end{aligned}
$$

Eq. (43) shows in a direct way the reduced effect of the chirality on the quasi-particle scattering rate in the presence of a sublattice inequivalence. For instance we can see that the back-scattering $\theta=\pi$ is now not completely suppressed but it is reduced by a factor $\Delta^{2} / \mu^{2}$ with respect to the forward-scattering $\theta=0$. For $|\mu|=\Delta$, when the chemical potential is on the edge of the gap, ${ }^{29}$ the $K_{1}$ angular dependence disappears completely and the quasi-particle scattering is completely isotropic with no effect of chirality.

Let us consider now the DC conductivity. Using the same derivation as in Sec. IV we can still write a set of equations as (30)-(32) with the same $c_{i}^{\alpha}, d_{i}^{\alpha}, f_{i}^{\alpha}$ and rescaled quantities $K_{0} \rightarrow \gamma_{\text {off }}^{2} K_{0}, K_{1} \rightarrow\left(1+\gamma_{z}^{2}\right) K_{1}$, $K_{2} \rightarrow \gamma_{\text {off }}^{2} K_{2}$, The presence of $\propto \hat{\sigma}_{z}$ terms in the Green's function gives rise in addition to a finite $z$-component $\Lambda_{z}^{\alpha}$. After lengthly but straightforward calculations we can still write the same self-consistent equation for the total vertex function as before, with the rescaling of the $K_{\alpha}$ coefficients discussed above. The final result is

$$
\Lambda_{\mathrm{RA}}^{\mathrm{tot}}(0)=\frac{\gamma_{\mathrm{off}}^{2} \Gamma_{\mathrm{qp}}}{\Gamma_{\mathrm{qp}}-\gamma_{\mathrm{off}}^{2} K_{0}-2\left(1+\gamma_{z}^{2}\right) K_{1}-\gamma_{\mathrm{off}}^{2} K_{2}},
$$

so that

$$
\begin{aligned}
\Gamma_{\mathrm{tr}} & =\frac{\Gamma_{\mathrm{qp}}-\gamma_{\mathrm{off}}^{2} K_{0}-2\left(1+\gamma_{z}^{2}\right) K_{1}-\gamma_{\mathrm{off}}^{2} K_{2}}{\gamma_{\text {off }}^{2}} \\
& =\frac{\mu^{2}+3 \Delta^{2}}{\mu^{2}-\Delta^{2}} K_{0}-\frac{4 \Delta^{2}}{\mu^{2}-\Delta^{2}} K_{1}-K_{2} .
\end{aligned}
$$

Eq. (44) looks at a first sight quite dim. However, taking into account the explicit angular dependence of the Eliashberg functions $\alpha^{2} F_{\alpha}$ and of the coefficients $K_{\alpha}$, we can re-arrange Eq. (44) in the form:

$$
\left\langle\Gamma_{\mathrm{tr}}\right\rangle_{\theta}=\left(1-\frac{\Delta^{2}}{\mu^{2}}\right)^{-1}\left\langle\Gamma_{\mathrm{qp}}(1-\cos \theta)\right\rangle_{\theta},
$$

where the presence of the angular term $(1-\cos \theta)$ suggests again the validity of the Boltzmann result even in this case where the chirality of graphene close to the Dirac point is affected by the sublattice inequivalence. As far as the prefactor is concerned, one could in principle justifies this as well within the Boltzmann framework by taking into account that the the opening of the gap affects also the Fermi velocity of the eigenstates of the Hamiltonian (38). Thus, following the Boltzmann prescription, the velocity in the basis of the eigenstates is $v_{k}=\hbar^{-1} d \sqrt{\left(\hbar v_{\mathrm{F}} k\right)^{2}+\Delta^{2}} / d k=v_{\mathrm{F}} \epsilon_{k} / \sqrt{\epsilon_{k}^{2}+\Delta^{2}} \approx$ $v_{F} \sqrt{1-\Delta^{2} / \mu^{2}}$, where $\epsilon_{k}=\hbar v_{\mathrm{F}} k$ and $\sqrt{\epsilon_{k}^{2}+\Delta^{2}} \approx \mu$ at the Fermi level. Thus, the additional $\left(1-\Delta^{2} / \mu^{2}\right)$ factor, that we obtained in Eq. (45) associated with vertex corrections, will appear in the Boltzmann expression for the conductivity $\sigma_{\mathrm{B}}=e^{2} N(\mu) v^{2} / 2\left\langle\Gamma_{B}\right\rangle$ associated to the $v^{2}$ term instead of the scattering rate, which is simply $\left\langle\Gamma_{\mathrm{B}}\right\rangle_{\theta}=\left\langle\Gamma_{\mathrm{qp}}(1-\cos \theta)\right\rangle_{\theta}$.

\section{DISCUSSION}

In the previous section we have shown that, in the limit where the chemical potential is the largest energy scale of the system, the Boltzmann theory is still valid for graphene even in the presence of a weak sublattice inequivalence which gives rise to a gap $\Delta$. A possible outcome of the extension of these calculations beyond this "Boltzmann regime" is suggested by the observation of the (weak) doping dependence of the resistivity $\rho$ that follows from Eq. (45). As it is shown in Fig. $7 \mathrm{a}$, both the low temperature $\left(T \ll \phi_{\max }\right)$ and the high temperature $\left(T \gg \varnothing_{\max }\right)$ regimes acquire a doping dependence, here parametrized in terms of the ratio $r=(\Delta / \mu)^{2}$. Particularly interesting appears the high-temperature regime where, using Eq. (44) and (A4), one finds:

$$
\rho=\frac{\mu^{2}+3 \Delta^{2}}{\mu^{2}-\Delta^{2}} \frac{\pi D^{2}}{4 \hbar e^{2} v_{\mathrm{F}}^{2} v_{s}^{2} \rho_{m}} T,
$$

which implies a significant increase of the linear slope as one approaches the Dirac point $\mu \rightarrow \Delta$, as shown in Fig. 7b. Observe that, if one would use instead the single-bubble result for the DC conductivity, the resistivity would be proportional to the quasiparticle scattering rate $\Gamma_{\mathrm{qp}}$, so that according to Eq. (43) one would obtain:

$$
\rho_{\text {bare }}=\left(1+\frac{\Delta^{2}}{\mu^{2}}\right) \frac{\pi D^{2}}{4 \hbar e^{2} v_{\mathrm{F}}^{2} v_{s}^{2} \rho_{m}} T,
$$

which has a much weaker doping dependence, as shown in Fig. 7b. Remarkably, an increase of the linear slope as $\mu$ decreases has been observed in recent measurements of resistivity in doped graphene samples. ${ }^{27,28}$ Indeed, while the measured crossover from a power-law to a linear $T$ behavior points toward the electron-phonon scattering as the source of the temperature dependence, the fact that the slope of the linear term decreases as doping increases, and eventually saturates at enough large doping, is unexplained within existing theories. Eq. (46) could suggest a possible mechanism for this doping dependence, 

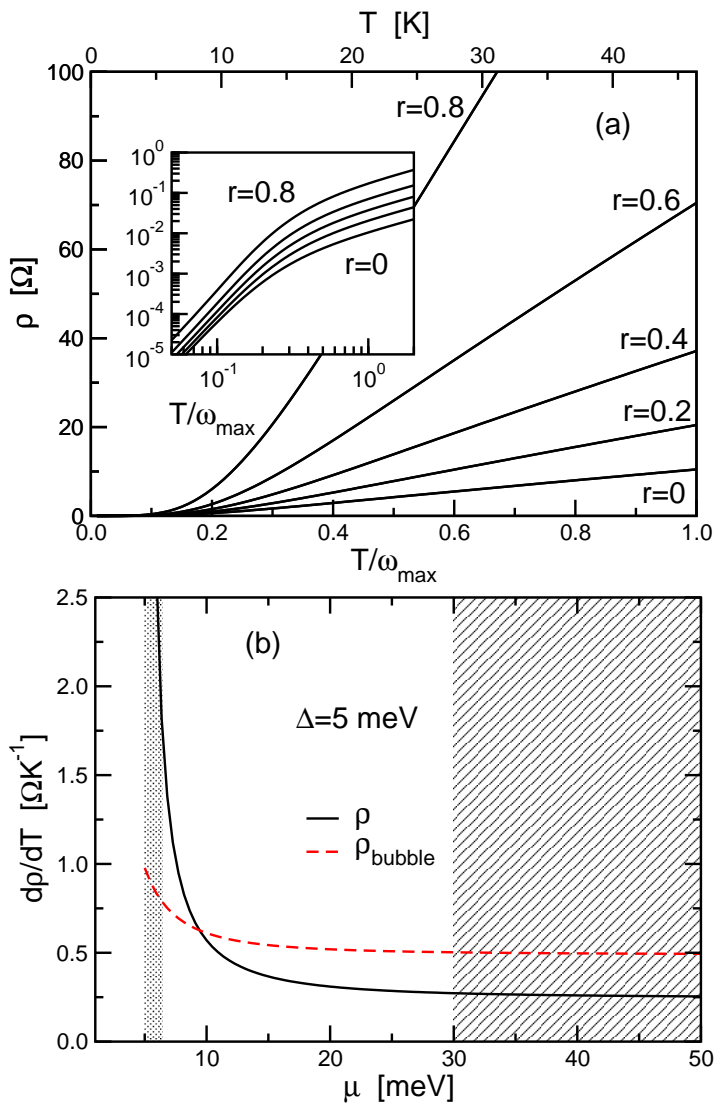

FIG. 7: (color online): (a) Temperature dependence of the resistivity for different doping in the presence of a weak sublattice inequivalence, giving rise to the gap $\Delta$. Here $r=\Delta^{2} / \mu^{2}$. Resistivity has been computed using the transport scattering time in Eq. (44). Inset: same quantities on a log-log scale. (b) Doping dependence of the high-temperature slope of the resistivity in graphene in the presence of a gap $(\Delta=5 \mathrm{meV})$. The dashed (red) line is the result (47) obtained with the bare bubble, while the solid (black) line represents the resistivity (46) computed with full inclusion of vertex corrections. The shaded area at $\mu>30 \mathrm{meV}$ represents the range of doping investigated in Ref. [28, while in the grey area around $5 \mathrm{meV}$ the validity of Eq. (46) fails because $|\mu|-\Delta \leq \Gamma_{\mathrm{qp}}\left(\Gamma_{\mathrm{qp}}=1.4\right.$ $\mathrm{meV}$ has been computed here for $T=300 \mathrm{~K}$ ).

even though in the regime of validity of this equation the quantitative variations of the slope are expected to be much smaller than what experimentally measured. Indeed, recent tunneling experiments performed in a similar suspended graphene sample have found a gap at the

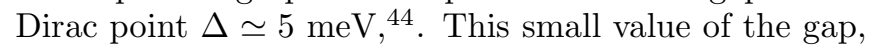
compared to the relevant values $\mu \simeq 30-70 \mathrm{meV}$ of the chemical potential in the samples investigated in Ref. 28, would lead, in Eq. (46), to a variation of the slope of only few percents, as shown in Fig. 7b. However, one should keep in mind that Eq. (46) has been derived in the limit $\Gamma_{\mathrm{qp}} \ll \mu$. Thus, only the full solution in the crossover region where the Dirac point is approached can discriminate if the doping dependence reported in Refs. 27,28 is an effect of a small gap opening which goes beyond the
Boltzmann approach. Analogously, it can be worth also to investigate alternative scenarios 45 for the gap opening that have been suggested by photoemission results in epitaxial graphene, $\stackrel{46.47}{ }$ where the gap $\Delta \simeq 100 \mathrm{meV}$ is comparable to the chemical potential in the interesting doping regime.

\section{CONCLUSIONS}

In summary, in this paper we outlined the issue of the calculation of the current vertex corrections for electronphonon scattering in graphene within the Kubo formalism. While previous works investigated the role of vertex corrections in the presence of impurity scattering, $\stackrel{24,25,26}{\underline{ }}$ here we address for the first time the issue of vertex corrections in graphene when the scattering mechanism arises from electron-phonon interactions, which have a non-trivial momentum dependence. We analyzed in particular the case of doped graphene, when $\mu$ is the largest energy scale of the system (i.e. $|\mu| \gg \omega_{\max }, \Gamma_{\mathrm{qp}}$ ). In this regime the calculations can be performed explicitly, leading to an analytical derivation of the transport scattering rate which appears in the DC conductivity. Remarkably, we found that despite the lack of direction-dependence of the quasiparticle velocity in graphene, the matrix structure associated to the current vertex plays a similar role than the momentum dependence of the renormalized vertex in ordinary metals, leading to a confirmation of the Boltzmann approach. Such a result is confirmed also in the presence of a sublattice inequivalence, which leads to a gapped Dirac spectrum and introduces a doping dependence of the resistivity that can be a promising candidate to explain existing experimental data in doped graphene samples. With respect to the formalism based on the quantum kinetic equations, $\stackrel{20,21,22}{=}$ which is also aimed to investigate the applicability of the Boltzmann result, the present approach based on the calculation of vertex corrections for the conductivity bubble has the main advantage of being suitable of a direct extension at finite frequency. Such an extension will lead to a full quantum treatment of the conductivity in graphene, and will be the subject of a future work.

\section{Acknowledgments}

We acknowledge financial support from MIUR under the Research Program MIUR-PRIN 2007.

\section{APPENDIX A: HARMONIC COMPONENTS OF THE ELIASHBERG FUNCTIONS AND $K_{\alpha}$ COEFFICIENTS}

In this Appendix we provide an explicit expression for the harmonic components of Eliashberg functions $\alpha^{2} F_{\alpha}(\Omega)$, and we calculate the temperature dependence 
of the corresponding functions $K_{\alpha}(T)$. We recall the definition of the momentum-dependent Eliashberg function,

$$
\alpha^{2} F\left(\mathbf{k}-\mathbf{k}^{\prime}, \Omega\right)=\left|g_{\mathbf{k}-\mathbf{k}^{\prime}}\right|^{2} \delta\left(\Omega-\omega_{\mathbf{k}-\mathbf{k}^{\prime}}\right),
$$

where $\omega_{\mathbf{q}} \approx \hbar v_{\mathrm{s}}|\mathbf{q}|$. Following Ref. 19, we notice that $\left|g_{\mathbf{q}}\right|^{2} \propto|\mathbf{q}|$, so that we can write $\left|g_{\mathbf{q}}\right|^{2}=I|\mathbf{q}|$, where $I=\hbar D^{2} / 2 V \rho_{m} v_{s}, D$ is the deformation potential, $V$ is the volume of the two-dimensional unit cell, and $\rho_{m}$ is the graphene mass density. Typical values are $D \simeq 19 \mathrm{eV}$, $\rho_{m}=7.6 \times 10^{-8} \mathrm{gr} / \mathrm{cm}^{2}, v_{s}=2 \times 10^{6} \mathrm{~cm} / \mathrm{s}, v_{\mathrm{F}}=10^{6} \mathrm{~m} / \mathrm{s}$. Restricting the electron momenta on the Fermi surface, $|\mathbf{k}| \approx\left|\mathbf{k}^{\prime}\right| \approx k_{\mathrm{F}}$, we can write $|\mathbf{q}|=2 k_{\mathrm{F}} \sin \left[\left(\phi-\phi^{\prime}\right) / 2\right]$, and the Eliashberg function will result to depend only on the exchanged angle $\theta=\phi-\phi^{\prime}$ :

$$
\alpha^{2} F(\theta, \Omega)=2 I k_{\mathrm{F}} \sin (\theta / 2) \delta\left[\Omega-\omega_{\max } \sin (\theta / 2)\right],
$$

where we remind $\omega_{\max }=2 \hbar v_{s} k_{\mathrm{F}}$.

The angular components $\alpha^{2} F_{\alpha}$ will be evaluated simply projecting the Eliashberg function (A1) on the spherical harmonics $\psi_{\alpha}(\phi)=\mathrm{e}^{i \alpha \phi}$. We have thus:

$$
\begin{aligned}
\alpha^{2} F_{\alpha}(\Omega) & =2 I k_{\mathrm{F}} \int_{0}^{2 \pi} \frac{d \theta}{2 \pi} \sin (\theta / 2) \mathrm{e}^{i \alpha \theta} \delta\left[\Omega-\omega_{\max } \sin (\theta / 2)\right. \\
& =2 I k_{\mathrm{F}} \int_{0}^{\pi} \frac{d \theta}{\pi} \sin \theta \mathrm{e}^{i 2 \alpha \theta} \delta\left[\Omega-\omega_{\max } \sin \theta\right]
\end{aligned}
$$

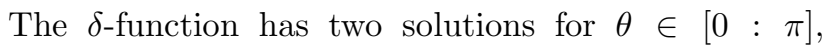
one for $\theta=y_{\Omega}$ and one for $\theta=\pi-y_{\Omega}$, where $y_{\Omega}=$ $\arcsin \left(\Omega / \omega_{\max }\right)$. We can write thus:

$$
\begin{aligned}
\alpha^{2} F_{\alpha}(\Omega)= & 2 I k_{\mathrm{F}} \int_{0}^{\pi} \frac{d \theta}{\pi} \sin \theta \mathrm{e}^{i 2 \alpha \theta} \frac{1}{\left|\omega_{\max } \cos (\theta)\right|} \\
& \times\left[\delta\left(\theta-y_{\Omega}\right)+\delta\left(\theta-\pi+y_{\Omega}\right)\right] \\
= & \frac{4 I k_{\mathrm{F}}}{\pi \omega_{\max }^{2}} \frac{\Omega \theta\left(\omega_{\max }-\Omega\right)}{\sqrt{1-\left(\frac{\Omega}{\omega_{\max }}\right)^{2}}} \\
& \times \frac{1}{2}\left[\mathrm{e}^{i 2 \alpha y_{\Omega}}+\mathrm{e}^{i 2 \alpha\left(\pi-y_{\Omega}\right)}\right] \\
= & \frac{2 I}{\pi \hbar v_{s}} \frac{\emptyset \cos \left(2 \alpha y_{\Omega}\right) \theta\left(\omega_{\max }-\Omega\right)}{\sqrt{\varnothing_{\max }^{2}-\emptyset^{2}}}
\end{aligned}
$$

where we made use of $\sin \left(y_{\Omega}\right)=\sin \left(\pi-y_{\Omega}\right)=\Omega / \omega_{\max }$, $\left|\cos \left(y_{\Omega}\right)\right|=\left|\cos \left(\pi-y_{\Omega}\right)\right|=\sqrt{1-\left(\Omega / \omega_{\max }\right)^{2}}$.

Inserting Eq. (A2) into the Eq. (16) which defines the $K_{\alpha}$ function, we can obtain their temperature dependence. By rescaling the integration variable to $y=\varnothing$ we have:

$$
\begin{aligned}
K_{\alpha} & =\frac{N(\mu) I}{\hbar v_{s}} \frac{T^{2}}{\emptyset_{\max }} \int_{0}^{\beta \omega_{\max }} d y \frac{y}{\sqrt{1-\left(y / \beta \omega_{\max }\right)^{2}}} \\
& \times \cos \left(2 \alpha \arcsin \frac{y}{\beta \omega_{\max }}\right)[n(y)+f(y)] .
\end{aligned}
$$

In the low-temperature limit $T \ll \varnothing_{\max }$ we can retain the leading terms in powers of $y / \beta \varnothing_{\max }$ in the integrand and send the upper limit of integration to infinity, $\beta \omega_{\max } \rightarrow$ $\infty$. We obtain thus:

$$
K_{\alpha}=\frac{N(\mu) I}{\hbar v_{s}} \frac{T^{2}}{\emptyset_{\max }}\left[a_{1}+a_{3} \frac{1-4 \alpha^{2}}{2} \frac{T^{2}}{\emptyset_{\max }^{2}}\right],
$$

where $a_{n}=\int_{0}^{\infty} d y y^{n}[n(y)+f(y)]$, and in particular, $a_{1}=$ $\pi^{2} / 4$ and $a_{3}=\pi^{4} / 8$. In the opposite high-temperature limit $T \gg \varnothing_{\max }$ we can instead compute the integrand function in Eq. (A33) as $y \rightarrow 0$. In this case, due to the rapid oscillations of the cosine term, all the harmonics vanish except the $\alpha=0$ one, which displays a linear $T$ dependence:

$$
K_{0}(T)=\frac{\pi N(\mu) I}{2 \hbar v_{s}} T
$$

\section{APPENDIX B: TOTAL QUASI-PARTICLE SCATTERING RATE IN THE PRESENCE OF SUBLATTICE INEQUIVALENCE}

In this Appendix we derive the effective total quasiparticle scattering rate in graphene in the presence of sublattice inequivalence.

Let us start from the scattering rates in Eqs. (40)-(42), which we can write in the matricial form:

$$
\hat{\Gamma}=\Gamma_{I} \hat{I}+\Gamma_{\text {off }}\left[\cos \phi \hat{\sigma}_{x}+\sin \phi \hat{\sigma}_{y}\right]+\Gamma_{z} \hat{\sigma}_{z}
$$

Considering the Hamiltonian in Eq. (38), we have then

$$
\begin{aligned}
\hat{G}^{-1}(\epsilon, \omega)= & \left(\hbar \omega+i \Gamma_{I}\right) \hat{I}+\left(\Delta-i \Gamma_{z}\right) \hat{\sigma}_{z} \\
& -\left(\epsilon-i \Gamma_{\text {off }}\right)\left[\cos \phi \hat{\sigma}_{x}+\sin \phi \hat{\sigma}_{y}\right] .
\end{aligned}
$$

The spectral function, whose width determines the total effective quasi-particle scattering rate, is associated with the $G_{I}$ term which results:

$$
G_{I}(\epsilon, \omega)=\frac{\hbar \omega+\mu+i \Gamma_{I}}{\left.\left(\hbar \omega+\mu+i \Gamma_{I}\right)^{2}-\left(\epsilon-i \Gamma_{\text {off }}\right)^{2}-\left(\Delta-i \Gamma_{z}\right)^{\frac{2}{2}}\right)}
$$

In order to identify the total effective quasi-particle scattering rate, we expand now the denominator and write, in particular,:

$$
\begin{aligned}
& \left(\epsilon-i \Gamma_{\text {off }}\right)^{2}+\left(\Delta-i \Gamma_{z}\right)^{2} \\
= & \epsilon^{2}+\Delta^{2}-\Gamma_{\text {off }}^{2}-\Gamma_{z}^{2}-2 i\left(\epsilon \Gamma_{\text {off }}+\Delta \Gamma_{z}\right) .
\end{aligned}
$$

Since $\epsilon^{2}+\Delta^{2} \approx \mu$ and $\Gamma_{\text {off }}, \Gamma_{z} \ll|\mu|$, we can neglect $\Gamma_{\text {off }}^{2}-\Gamma_{z}^{2}$ with respect to $E^{2}=\epsilon^{2}+\Delta^{2}$ and, at the same order, we can write:

$$
\begin{aligned}
& E^{2}-2 i\left(\epsilon \Gamma_{\text {off }}+\Delta \Gamma_{z}\right) \approx\left[E-i\left(\frac{\epsilon}{E} \Gamma_{\text {off }}+\frac{\Delta}{E} \Gamma_{z}\right)\right]^{2} \\
\approx & {\left[E-i\left(\sqrt{1-\frac{\Delta^{2}}{\mu^{2}}} \Gamma_{\text {off }}+\frac{\Delta}{\mu} \Gamma_{z}\right)\right]^{2} }
\end{aligned}
$$


Plugging this result in (B2), we can now split the resulting Green's function in the usual two contributions from the upper and lower band,

$$
G_{I}(E, \omega)=\frac{1}{2} \sum_{s= \pm} \frac{1}{\hbar \omega+\mu+i \Gamma_{I} \mp E \pm i \Gamma_{ \pm}}
$$

with

$$
\Gamma_{ \pm}=\Gamma_{I} \pm\left(\sqrt{1-\frac{\Delta^{2}}{\mu^{2}}} \Gamma_{\text {off }}+\frac{\Delta}{\mu} \Gamma_{z}\right)
$$

${ }^{1}$ F. Guinea, N.M.R. Peres, K.S. Novoselov, and A.K. Geim, arXiv:0709.1163 [cond-mat] (2007).

2 T. Ando and T. Nakanishi, J. Phys. Soc. Jpn 67, 1704 (1998).

3 P.A. Lee, Phys. Rev. Lett. 71, 1887 (1993)

4 P.J. Hirschfeld, W.O. Putikka, and D.J. Scalapino, Phys. Rev. B 5010250 (1994).

5 Y.S. Lee, K. Segawa, Z.Q. Li, W.J. Padilla, M. Dumm, S.V. Dordevic, C.C. Homes, Y. Ando, and D.N. Basov, Phys. Rev. B 72054529 (2005).

${ }^{6}$ E. McCann and V.I. Fal'ko, Phys. Rev. Lett. 96, 086805 (2006).

7 T. Holstein, Ann. Phys. 29, 410 (1964).

8 G.D. Mahan, Many-Particle Physics (Plenum, New York, 1981).

9 K. Nomura and A.H. MacDonald, Phys. Rev. Lett. 96, 256602 (2006).

10 T. Ando, J. Phys. Soc. Jpn 75, 074716 (2006).

11 E.H. Hwang, S. Adam, and S. Das Sarma, Phys. Rev. Lett. 98, 186806 (2007).

12 S. Adam, E.H. Hwang, V. Galitski, and S. Das Sarma, Proc. Natl. Acad. Sci. USA 104, 18392 (2007).

13 D.S. Novikov, Phys. Rev. B 76, 245435 (2007).

14 T. Stauber, N.M.R. Peres, and F. Guinea, Phys. Rev. B 76, 205423 (2007).

15 N.M.R. Peres, J.M.B. Lopes dos Santos, T. Stauber, Phys. Rev. B 76, 073412 (2007).

16 S. Adam and S. Das Sarma, Phys. Rev. B 77, 115436 (2008).

17 S. Adam and S. Das Sarma, Solid State Communications 146, 356 (2008).

18 E.H. Hwang and S. Das Sarma, Phys. Rev. B 77, 195412 (2008).

19 E.H. Hwang and S. Das Sarma, Phys. Rev. B 77, 115449 (2008).

${ }^{20}$ M. Auslender and M.I. Katsnelson, Phys. Rev. B 76, 235425 (2007).

21 M. Trushin and J. Schliemann, Phys. Rev. Lett. 99, 216602 (2007).

22 M. Trushin and J. Schliemann, Europhys. Lett. 83, 17001 (2008).

23 J.M. Ziman, G.D. Mahan, Electrons and Phonons (Oxford University Press, New York, 1960).

24 N.H. Shon and T. Ando, J. Phys. Soc. Jpn 67, 2421 (1998).

25 E. McCann, K. Kechedzhi, V.I. Fal'ko, H. Suzuura, T. Ando, and B.L. Altshuler, Phys. Rev. Lett. 97, 146805 (2006).

${ }^{26}$ K. Kechedzhi, O. Kashuba, and V.I. Fal'ko, Phys. Rev. B$$
\Gamma_{\mathrm{qp}}=\Gamma_{I}+\sqrt{1-\frac{\Delta^{2}}{\mu^{2}}} \Gamma_{\mathrm{off}}+\frac{\Delta}{\mu} \Gamma_{z}
$$

so that at the Fermi level in the upper band we obtain

77, 193403 (2008).

27 J.H. Chen, C. Jang, S. Xiao, M. Ishigami, and M.S. Fuhrer, Nat. Nanot. 3, 206 (2008)

28 K.I. Bolotin, K.J. Sikes, J. Hone, H.L. Stormer, and P. Kim, Phys. Rev. Lett. 101, 096802 (2008).

29 M. Mucha-Kruczyński, O. Tsyplyatyev, A. Grishin, E. McCann, V.I. Fal'ko, A. Bostwick, and E. Rotenberg, Phys. Rev. B 77, 195403 (2008).

30 A. Bostwick, T. Ohta, J.L. McChesney, K.V. Emtsev, T. Seyller, K. Horn, and E. Rotenberg, New. Jour. of Phys. 9, 385 (2007).

31 Z.Q. Li, E.A. Henriksen, Z. Jiang, Z. Hao, M.C. Martin, P. Kim, H.L. Stormer, and D.N. Basov, Nat. Phys. 4, 532 (2008).

32 A.H. Castro Neto and F. Guinea, Phys. Rev. B 75, 045404 (2007).

33 Ge.G. Samsonidze, E.B. Barros, R. Saito, J. Jiang, G. Dresselhaus, and M.S. Dresselhaus, Phys. Rev. B 75, 155420 (2007).

34 J.L. Mañes, F. Guinea, and M.A.H. Vozmediano, Phys. Rev. B 75, 155424 (2007).

35 C.-H. Park, F. Giustino, M.L. Cohen, and S.G. Louie, Phys. Rev. Lett. 99, 086804 (2007).

36 N. Bonini, M. Lazzeri, N. Marzari, and F. Mauri, Phys. Rev. Lett. 99, 176802 (2007).

37 M. Calandra and F. Mauri, Phys. Rev. B 76, 205411 (2007).

38 W.-K. Tse and S. Das Sarma, Phys. Rev. Lett. 99, 236802 (2007).

39 D.M. Basko and I.L. Aleiner, Phys. Rev. B 77, 041409 (2008).

40 A.B. Migdal, Zh. Eksp. Teor. Fiz. 34, 1438 (1958) [Sov. Phys. JETP 7, 996 (1958)].

41 G.M. Eliashberg, Zh. Eksp. Teor 38, 966 (1960) [Sov. Phys. JETP 11, 696 (1958)].

42 V.P. Gusynin, S.G. Sharapov, and J.P. Carbotte, Phys. Rev. Lett. 96, 256802 (2006).

43 V.P. Gusynin, S.G. Sharapov, and J.P. Carbotte, Int. J. Mod. Phys. B 21, 4611 (2007).

44 G. Li, A. Luican, and E.Y. Andrei, arXiv:0803.4016.

45 L. Benfatto and E. Cappelluti, Phys. Rev. B 78, 115434 (2008).

46 S. Y. Zhou, G. -H. Gweon, A. V. Fedorov, P. N. First, W. A. de Heer, D. -H. Lee, F. Guinea, A. H. Castro Neto, and A. Lanzara, Nat. Mat. 6, 770 (2007).

47 S.Y. Zhou, D.A. Siegel, A.V. Fedorov, and A. Lanzara, Physica E 4, 2642 (2008). 